\title{
QUINTILIANO Y LA SEMIÓTICA DEL TEATRO
}

Recent research on the Semiotics of theatre derives its theoretical and methodological assumptions from Czech structuralism and from the studies of the Polish semiotician T. Kowzan. Although there is no evidence of Ancient theories on the theatrical performance, the analysis of Quintilian's rhetorical texts leads, through analogy with rhetorical practice, to the conclusion that many of the concepts so far used had already been formulated by theoreticians in ancient times.

Siguiendo una línea de recuperación del pensamiento lingüistico de la antigüedad, pretendemos poner de relieve una injusta e injustificable ausencia en lo que hoy día se conoce como Semiótica Teatral, disciplina estrechamente unida al nombre del investigador polaco Tadeusz Kowzan y a los estudios realizados a partir de los años sesenta, con antecedentes en el llamado Círculo de Praga '.

Aunque el interés de la Semiótica moderna por conceptos tales como los de "semiotización de la escena», "connotación» y "movilidad del signo teatral», o la cada vez mayor importancia concedida al análisis de la "puesta en escena", se hace derivar de la obra de Kowzan, esta afirmación, sin embargo, no parece del todo acertada. Pues, si bien es cierto que no ha llegado hasta nosotros ningún escrito teórico sobre la representación teatral en la antigüedad, de hecho nos encontramos con abundantes noticias sobre obras dedicadas a analizar aspectos concretos

1 T. Kowzan, "The sign in the theater", Diogenes 61,1968 , pp. 52-80. Sobre los inicios de la Escuela de Praga cf. L. Matejka e I. Titunik (edd.), Semiotics of Art: Prague School Contributions, Cambridge, Mass., 1976; F. Deak, "Structuralism in theatre: Prague School Contribution", The Drama Review 20, 1976, pp. 83-94; I. Slavinska, "La semiologia del teatro in statu nascendi: Praga 1931-1941", Biblioteca teatrale 1, 1978, pp. 114-135; K. Elam, The Semiotics of Theatre and Drama, Londres 1980, pp. 5-19; una reciente valoración en T. Sebeok (ed.), Encyclopedic Dictionary of Semiotics, Berlin 1986, II, s.u. "theater». 
de la práctica del teatro como la pronunciación, los movimientos o los gestos de los actores. Por otra parte, contamos también con la ayuda que nos proporciona la Retórica, en particular con lo que podriamos definir como la "puesta en escena" oratoria, esto es, con la denominada dictio, en la que se engloban actio y pronuntiatio. Prueba de ello son las frecuentes referencias en los escritos retóricos a la forma de actuar de los oradores en comparación con la de los actores, hecho que permitiría asumir la hipótesis de la existencia en la antigüedad de una "teoría general de la presentación" aplicable a diferentes dominios.

Nos interesa especialmente destacar esto último ya que, en contra de lo que se suele opinar en la actualidad, no creemos que la consideración que juega la "representación» en el hecho teatral constituya un descubrimiento moderno ${ }^{2}$. Cierto es que Aristóteles había afirmado que el espectáculo, en tanto que ajeno al arte de la escritura, no es competencia de la poesía; sin embargo, consideramos imprescindible situar estas palabras en el contexto de su Poética, cuyo interés está centrado en el texto literario y en la actividad del escritor. Desde esta perspectiva la afirmación de Aristóteles es perfectamente comprensible. Aun así, no es menos cierto que también señala cómo para la puesta en escena de una obra dramática es más importante el arte de la escenografia que el del poeta, y - añade - «ya que la imitación se realiza por medio de personajes que actúan, necesariamente una parte esencial de la tragedia ha de ser el buen efecto del espectaculo" ${ }^{3}$.

Por otra parte, sabemos, al menos, de la existencia de las obras de dos autores, Nigidio Fígulo y Plocio, que escribieron sobre el gesto en los actores y en los oradores, aproximando asi la práctica de ambos grupos profesionales ${ }^{4}$. Por último, es ampliamente reconocido que la Retórica llegó a constituir en determinados aspectos - específicamente en lo que se refiere a la dictio - una auténtica «teoría de la representación». El paralelismo mencionado entre actores y oradores viene avalado no sólo por los estudios de filólogos ${ }^{5}$, o de semióticos ${ }^{6}$, sino sobre

${ }^{2}$ Sobre la reivindicación del espectáculo véase T. Sebeok, o.c., y P. Pavis, Dictionnaire du théâtre. Termes et concepts de l'analyse théâtrale, Paris 1980, s.u. "Texte dramatique», "texte scenique" y "texte et scène".

${ }^{3}$ Cf. Arist., Poet. 1449-50a, ed. R. Kassel, Oxford 1965. Sobre la noción de "puesta en escena" y su modernidad, cf. P. Pavis, o.c., s.u. «mise en scène».

4 Quint., I.O. XI 3,143; cf. los estudios de K. Ziegler, "L. Plotius Gallus», RE XXI (1951), cols. 598-601, y G. Perl, «Die Stellung der Latini rhetores innerhalb der römischen Rhetorik", Acta Antiqua Philoppopolitana, 1962, Sofia 1963, pp. 265-284. Sobre Plocio, véase Quint., I.O. II 4,42 y el comentario de J. Cousin en su edición del libro II, París, Les Belles Lettres, pp. 238-239.

5 Cf. B. A. Taladoire, Commentaire sur la mimique et l'expression corporelle du comédien romain, Montpellier 1951, p. 88 ss.; J. W. Basore, "Quintilian on the status 
todo y fundamentalmente, por los propios oradores y rétores ${ }^{7}$, quienes, al presentar las normas para la práctica oratoria, recurrían continuamente a la comparación con el modo de hacer de los actores y con el espectáculo teatral.

En esta línea, intentaremos mostrar la relevancia de Quintiliano en este campo, objetivo para el que nos apoyaremos especialmente en el capítulo 3 del libro XI de su Institutio Oratoria, dedicado a las partes de la dictio, o sea a la actio y a la pronuntiatio.

Aunque actualmente no es posible valorar en su justa medida la importancia de esta parte de la Retórica, y en cierto modo se acostumbra a menospreciarla, todos los escritos de los rétores que conservamos coinciden unánimente en subrayar la relevancia de este aspecto en la enseñanza retórica ${ }^{8}$. Y estos dos términos -actio y pronuntiatio-, para muchos referidos a un mismo objeto, vuelven a señalar la ya mencionada estrecha relación entre actores y oradores. Así actio designa en el lenguaje jurídico la "acción oratoria», y el sustantivo agente actor tiene el sentido de «abogado», en tanto que en la lengua del teatro, desde Plauto ${ }^{9}$, estos mismos términos se emplean para designar respectivamente a la "representación" y al "actor». Junto a esto, pronuntiatio traduce el griego únóxpıðıs, relacionado con la denominación del actor

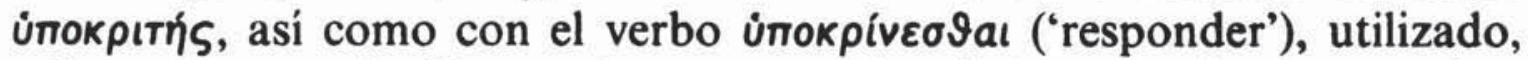
sobre todo, en relación con la respuesta de adivinos y oráculos, uso éste del que pasó al significado de 'dar la réplica' $y$, finalmente, al de 'representar un papel', según se puede concluir de la comparación de los textos de Aristófanes y Aristóteles ${ }^{10}$.

En la obra de Quintiliano, actio y pronuntiatio son usados un número casi igual de veces; $y$, aunque el primero de ellos parece reservado al "gesto" y el segundo a la "voz" (pronuntiatio a plerisque actio dicitur, sed prius nomen a uoce, sequens a gestu uidetur accipere, I.O. XI 3,1), de

of the later comic stage", TAPhA 40, 1909, pp. 21-22; P. Grimal, "Le rôle de la mise-en-scène dans les tragédies de Sénéque. Clytemnestra et Cassandre dans l'Agammenon", en Théâtre et spectacles dans l'Antiquité, Estrasburgo 1981, pp. 123139; A. Pociña, "Quintiliano y el teatro latino", CFC 17, 1981-82, pp. 97-110.

"Cf. F. Ruffini, "Pseudosemiotica, pseudoteatro e presentazioni», Biblioteca teatrale 20, 1978, y A. Saraiva, "Message et Littérature», Poétique 5, 1974, pp. 1-13.

${ }^{7}$ Las principales referencias en Cicerón y Quintiliano se encuentran en Brut. 290; De orat. V 18 y II 338; I.O. I 2,1; II 10,13; X 1,46-131; X 1,65 y 69; XI 3,4, y XI 3,74 .

${ }^{8}$ Véanse Cic., De orat. III 56, 213; Orat. 55-56; Brut. 110; De orat. III 59,224, y Quint., I.O. XI 3,2.

${ }_{9}$ Plaut., Bacch. 213 non res, sed actor mihi cor odio sauciat.

${ }^{10}$ Aristóf., Vesp. 52, y Arist., Rhet. 1403b 23. Sobre el término en griego, B. Luccheli, 'Ynokpırís. Origine e storia del termine, Génova 1962. 
hecho en la práctica les atribuye idénticos dominios (utraque appellatione indifferenter uti licet, ibid.) ". En lo que aquí nos atañe, interesa destacar la importancia concedida por el rétor a esta parte de la Retórica, tanto por su "fuerza" como por su "eficacia», ya que - señala - tam refert qualia sint quae intra nosmet ipsos composuimus quam quo modo efferantur (I.O. XI 3,2), esto es, importa menos lo que hayamos compuesto que la manera en que lo exponemos. Destaca así Quintiliano el fundamental papel que juega la "puesta en escena oratoria», consciente de la capacidad de seducción de la palabra en función de la habilidad a la hora de la exposición, llegando incluso a recordar los efectos sobre el destinatario de la comunicación: Nam ita quisque, ut audit, mouetur. Quare neque probatio ulla, quae modo uenit ab oratore, tam firma est ut non perdat uires suas, nisi adiuuatur adseueratione dicentis (ibid.) ${ }^{12}$, opinión ésta que no limita exclusivamente al uso de la voz por parte del orador, sino que hace extensiva a la fisonomía y a la actitud corporal del hablante ${ }^{13}$.

Con esto plantea Quintiliano cuestiones tales como la relevancia de la representación, el efecto de ésta sobre el auditorio, la aparición de distintos tipos de $\operatorname{signos}^{14}$, y, en definitiva, la coexistencia de diferentes sistemas significantes ${ }^{15}$, problemas que se aproximan extraordinariamente a temas estudiados como novedad por la reciente Semiótica Teatral. Con otras palabras reconoce el rétor lo que desde principios de siglo se conoce bajo la denominación de "teatralidad" ${ }^{16}$, concepto en el cual se incluyen tres rasgos fundamentales que, como veremos, se encuentran ya esbozados y tratados - a veces con un increíble detallepor el autor latino.

Un primer rasgo está referido a la «heterogeneidad» del conjunto de sistemas significantes implicados en la representación -oratoria o tea-

"Sobre el sentido de los términos y su empleo indiscriminado cf. Cic., Orat. 55; De orat. III 222; pronuntiatio en Rhet. ad Her. I 3 y III 19; Cic., Inu. I 9; actio prevalece en De orat., Brut., Orat., Opt. gen. y Part.; actio igual a pronuntiatio en Quint., I.O. XI 3,3.

12 Véase asimismo XI 3,153.

${ }^{13}$ Cf. Cic., De orat. II 190.

14 Cf. I.O. XI 3,14 Cum sit autem omnis actio, ut dixi, in duas diuisa partis, uocem gestumque, quorum alter oculos, altera aures mouet, per quos duos sensus omnis ad animum penetrat adfectus...

is Sobre el concepto de sistema, K. Elam, The Semiotics of Theatre and Drama, Londres 1980, pp. 49-50; U. Eco, $A$ theory of Semiosics, Bloomington, Indiana (trad. esp., Barcelona 1977), y "Semiotics of theatrical Performance», The Drama Review 21, 1977, pp. 107-117.

16 Véase N. Eureïnoff, Histoire du Théâtre russe, cap. 10, París, Chêne, y J. Jachymiak, "Sur la théâtralité», Litterature-Science-Ideologie, 1972, pp. 49-58. 
tral - (gesto, palabras, decorado, etc.), cualidad ésta mediante la cual se opone la "puesta en escena" a la "homogeneidad» organizada a través de los textos literarios ${ }^{17}$. Este aspecto está claramente expresado en un pasaje en el que explícitamente se subraya la necesidad de cooperación y la interrelación entre los signos y sistemas que intervienen en la actio: Adfectus omnes languescant necesse est, nisi uoce, uultu, totius prope habitu corporis inardescunt $\left(\right.$ I.O. XI 3,2) ${ }^{18}$.

El segundo rasgo recoge la "espacialidad» de la puesta en escena (actio), esto es, su consideración como lenguaje espacial y temporal, algo ya sugerido por Cicerón ${ }^{19}$ y que corrobora Quintiliano al definir esta parte de la Retórica como un auténtico lenguaje, según veremos a continuación. Corolario de la "espacialidad» es la "simultaneidad» de los signos que participan en el proceso comunicativo de la «representación", aspecto éste que tampoco escapó a la atención de Quintiliano como se deduce del siguiente texto: Nec mirum si ista, quae tamen in aliquo posita sunt motu, tantum in animis ualent, cum pictura, tacens opus et habitus semper eiusdem, sic in intimos penetret adfectus ut ipsam uim dicendi nonnumquam superare uideatur. Contra si gestus ac uultus ab oratione dissentiat, tristia dicamus hilares, adfirmemus aliqua renuentes, non auctoritas modo uerbis, sed etiam fides desit (I.O. XI 3,67) ${ }^{20}$.

El tercer rasgo se refiere a lo que Barthes ${ }^{21}$, en su acertada y conocida definición del teatro, denominó "polifonía», término con el que designó la pluralidad simultánea de los mensajes (el espesor de los signos), cualidad que hace de la representación oratoria o teatral un fenómeno informativo diferente y opuesto a la monodia literaria. De este carácter también Quintiliano era consciente como muestra en repetidos pasajes al referirse a la pronuntiatio, así en el más arriba citado: adfectus omnes languescant..., o cuando recuerda la necesidad de cooperación de todos los sistemas implicados: Et hercule cum ualeant multum uerba per se et uox propriam uim adiciat rebus et gestus motusque significet aliquid, profecto perfectum quiddam fieri cum omnia coierunt necesse est (I.O. XI $3,9)^{22}$.

En este punto nos interesa destacar de un modo particular las palabras con las que apoya la relevancia de la actio, y ello por dos motivos. En primer lugar, porque recurre a la comparación explícita con la pues-

\footnotetext{
17 J. Jachymiak, o.c., p. 53.

18 Cf. igualmente Cic., De orat. II 190.

19 De orat. III 222 y Orat. 55.

${ }^{20}$ Cf. ibid. XI 65-68 y VI 2,35.

${ }^{21}$ R. Barthes, Essais Critiques, París 1964, p. 2.

22 Cf. I.O. XI 3,67, y Cic., De orat. III 57, 216.
} 
ta en escena teatral ${ }^{23}$, reforzando así el citado paralelismo entre actividad oratoria y práctica teatral, y, en segundo lugar, porque resume uno de los problemas centrales con los que se ha enfrentado la Semiótica teatral: la oposición texto/escena ${ }^{24}$. Especialmente significativo a este respecto nos parece lo que este autor escribe: Documentos sunt uel scaenici actores, qui et optimis poetarum tantum adiciunt gratiae, ut nos infinite magis eadem illa audita quam lecta delectent, et uilissimis etiam quibusdam impetrant aures, ut, quibus nullus est in bibliothecis locus, sit etiam frequens in theatris (I.O. XI 3,4) ${ }^{25}$. Es evidente que Quintiliano, mucho antes que los teóricos del Círculo de Praga, reconoce la finalidad de los textos dramáticos y por esta razón otorga una importancia capital a la "puesta en escena», llegando incluso a situarla por encima del texto escrito, idea que en la actualidad defienden como novedad algunos semióticos dedicados al estudio del teatro. Hay que recordar por otra parte que el destacado papel concedido a la actio (y/o pronuntiatio) es perfectamente comprensible si tenemos presente que muchas acciones judiciales llegaban a constituir acontecimientos con carácter de auténtico espectáculo, baste recordar el que nos describe Cicerón en su Pro Murena ${ }^{26}$.

Antes de detenernos en el análisis de los diferentes elementos que Quintiliano distingue y estudia en la "puesta en escena oratoria", conviene, aunque sea brevemente, hacer mención de las ideas que tiene el rétor sobre el concepto de signo. Para esta tarea son en particular útiles las consideraciones que lleva a cabo en el libro V de la Institutio Oratoria, observaciones que, como en toda la tradición retórica que se extiende desde Aristóteles hasta Cicerón, se encuentran fuertemente condicionadas por un enfoque predominantemente jurídico y forense.

Los signa, como los exempla o los argumenta, están en íntima vinculación con las probationes artificiales ${ }^{27}$, esto es, son «evidencias» en el sentido jurídico, que el ars del orador obtiene para procurar la absolución o la condena del acusado; no se debe olvidar, no obstante, que,

${ }^{23}$ Las alusiones a la actividad teatral son muy frecuentes, cf. nota 7 e I.O. I 2,1; II 10,$13 ;$ X 1,46-131; X 1,65-69; XI 3,74.

${ }^{24}$ Sobre la antítesis texto/escena, cf. P. Pavis, «Remarques sur le discours théâtral», Degrés 13, 1978, pp. 1-10, y Dictionnaire du théâtre, París 1980, s.u. "sémiologie théatrale»; M. De Marinis, "Lo spettacolo come testo, II», Versus 22, 1979, pp. 3-31, y A. Ubersfeld, Lire le théâtre, París 1978.

${ }_{25}$ Véase ibid. XI 3,7.

${ }^{26}$ Cic., De orat. II 338 y III 220; Brut. 224 y Orát. 138.

27 I.O. V 9,1 Omnis igitur probatio artificialis constat aut signis aut argumentis aut exemplis; cf. ibid. V 8 de probatione artificiali, V 9,1-14 de signis, V 10,1-125 de argumentis; V 11,1-44 de exemplis. 
junto a la orientación jurídica, la retórica de este autor está también dirigida a la consideración téorica y lógica. En términos generales, los signa son concebidos como antecedentes que conducen a consecuentes y, puesto que su punto de vista es esencialmente aristotélico y no estoico, el rétor considera los signos como «hechos» o «cosas», y no como proposiciones, este hecho es particularmente interesante dado que le permite también tener en cuenta los signos no verbales, de tanta trascendencia en la representación teatral y en la actio oratoria.

$\mathrm{Al}$ estar encaminado su interés fundamentalmente hacia el aspecto informativo de los signos, establece una primera distinción entre aquellos que sirven para lograr una información cierta y aquellos otros que producen tan sólo una información probable, es decir, entre signa necessaria y signa non necessaria. Los primeros, definidos como quae aliter habere se non possunt (I.O. V 9,3), son signos «inseparables», esto es, en los que el antecedente se encuentra estrechamente unido a su consecuente, y se corresponderían con lo que la actual teoría semiótica recoge bajo la denominación de "señales», signos a partir de los cuales es posible deducir con seguridad cierto estado de $\operatorname{cosas}^{28}$. La segunda clase de signos, los «no necesarios», producen información menos fiable al estar basados en la communis opinio, o sea, en normas de carácter estadístico o en convenciones sociales, y no en fundamentos científicos sólidos. Éstos, denominados sin mayor precisión por Quintiliano signa, uestigia o indicia, ${ }^{29}$ son, como hemos dicho anteriormente, signos en los que no existe una relación de necesidad entre antecedente y consecuente, y resultan particularmente importantes para nuestro propósito en la medida en que bajo esta categoría son clasificados actos o propiedades inherentes a los sujetos que pueden ser traducidos a proposiciones, pero que no ofrecen conocimiento seguro al estar basados tan sólo en verosimilitudes.

Sin ánimo de leer en Quintiliano más de lo que realmente escribió, en su clasificación de los signos encontramos un atisbo de la distinción que los semióticos establecen entre "comunicación» y «significación», o, más exactamente, entre «señales» e «indicios». Por otra parte, este análisis presenta una extraordinaria similitud con las teorías del que ha sido llamado fundador de la Semiótica Teatral, T. Kowzan, quien diferencia entre "signos naturales» y "signos artificiales», de manera análoga a la planteada por el rétor latino al referirse al gesto ${ }^{30}$. Efectivamen-

${ }^{28}$ U. Eco, Tratado de Semiótica general, o.c., p. 55.

29 I.O. V $9,9$.

${ }^{30} \mathrm{Cf}$. I.O. XI 3,88 Et hi quidem de quibus sum locutus cum ipsis uocibus naturaliter exeunt; alii sunt qui res imitatione significant. 
te el investigador polaco señala que en los signos naturales, determinados únicamente por las leyes fisicas, significante y significado están unidos por una relación de causa-efecto, en tanto que los signos artificiales dependen de la intervención humana; sin embargo, la oposición entre ambos tipos de signos no es absoluta, ya que con frecuencia los signos naturales exigen el acto motivado de inferir la relación entre significante y significado. Precisamente de este hecho se vale Kowzan para formular el concepto de "artificialización" de los signos naturales puestos en escena, señalando algo que ya se vislumbra en Quintiliano a juzgar por los detallados y minuciosos consejos que da al futuro orador: que los signos que emplea el arte teatral pertenecen todos a la categoría de los signos artificiales, en tanto que son consecuencia de un proceso voluntario, casi siempre creado con premeditación y con la finalidad de comunicar instantáneamente ${ }^{31}$.

Al igual que T. Kowzan, o más recientemente E. Fischer-Lichte ${ }^{32}$, Quintiliano estructura los signos que intervienen en la actio en función del órgano receptor, esto es, según sean visuales o acústicos ${ }^{33}$. A este último canal va dirigido lo que el rétor denomina uox, término bajo el cual cabe recoger los dos primeros sistemas que Kowzan separa en el continuum de la representación, a saber, la "palabra» y el "tono», así como lo que para E. Fischer-Lichte son signos «lingüisticos» y "paralingüísticos». El segundo elemento que distingue el rétor, el gestus, es equiparable a los signos que en el semiótico polaco están recogidos como sistemas mímico, gestual y proxémico, y que la investigadora alemana engloba bajo la denominación común de signos cinésicos ${ }^{34}$.

Con respecto a la uox, aunque se inscribe en la tradición retórica anterior, Quintiliano desarrolla sus ideas con un detalle y una similitud con el modo de actuar de los semióticos actuales que no deja de resultar sorprendente. Diferencia así, por ejemplo, entre la naturaleza de la voz y su empleo ${ }^{35}$, y a continuación, con una riqueza de vocabulario que incluso dificulta considerablemente la traducción, aconseja sobre aspectos como la «articulación», la «entonación», la «modulación», la "dura-

${ }^{31}$ T. Kowzan, o.c., p. 34.

${ }^{32}$ Cf. E. Fischer-Lichte, Semiotik des Theaters, vol.I, Das System der theatralischen Zeichen, Tübingen 1983.

${ }^{33} \mathrm{Cf}$. I.O. XI 3,17. La distinción entre uocis figura y corporis motus se remonta probablemente a Teofrasto, véase Anon. IV, 35,31 Waltz. Cicerón habla de uox, gestus y uultus, aunque establece una unión entre gestus y uultus, cf. Orat. 55, y A. Desmouliez, Cicéron et son goût, Latomus, Bruselas 1976.

${ }^{34}$ La separación en el ámbito del gesto de lo que atañe exclusivamente al rostro ya está en Cicerón, De orat. III 216.

${ }^{35}$ I.O. XI 3,14. 
ción», la «intensidad» o la «altura» ${ }^{36}$. En este punto no hemos podido dejar de comparar sus reflexiones con el modelo de análisis de las emociones propuesto por el psicólogo E. Davitz ${ }^{37}$, como resultado de lo cual constatamos que el rétor no deja de tener en cuenta ninguno de los factores básicos que en el autor citado encontramos, y ello no con una menor coherencia.

Así, mucho antes de que se formulara el concepto de rasgos paralingüísticos, escribe Quintiliano: Illud uero maximum..., quod secundum rationem rerum de quibus dicimus animorumque habitus conformanda uox est, ne ab oratione discordet (I.O. XI 3,45). Igualmente al referirse a la forma apropiada de pronuntiatio, señala: quae certe ea est quae iis de quibus dicimus accommodatur. Quod quidem maxima ex parte praestant ipsi motus animorum sonatque uox ut feritur (I.O. XI 3,61). La voz, añade, debe ser: sic uelut media uox, quem habitum a nostris acceperit, hunc iudicum animis dabit: est enim mentis index ac totidem quot illa mutationes habet $\left(\right.$ I.O. XI 3,62) ${ }^{38}$. Para apreciar la novedad del tratamiento que esto supone basta recordar la observación de Kowzan de que, en el teatro, la palabra no es sólo signo lingüístico, ya que también la forma en que se pronuncia le otorga valores complementarios. En este sentido, hay que mencionar que la clasificación ${ }^{39}$ que hizo este estudioso incluye bajo la denominación general de «tono» una serie de elementos como «entonación», «ritmo", "velocidad», «intensidad de acento», etc..., que resulta mucho menos completa y coherente que la ordenación establecida por Quintiliano. Puede añadirse asimismo que los modernos estudios paralingüísticos ${ }^{40}$ han identificado diversas clases de hechos vocálicos que ya aparecen claramente tratados por el rétor latino, así, por ejemplo, los derivados de factores físicos como puedan ser la edad o el sexo.

A este respecto, la investigación de las últimas décadas ha puesto de manifiesto, por una parte, que la conducta paralingüística es adquirida mediante un proceso de aprendizaje, y, por otra, que es específica de cada cultura, hasta el punto de que resulta posible explicar y justificar no sólo los tipos, sino también las funciones - expresivas, emotivas, etc...- de la actividad vocálica de los hablantes ${ }^{41}$. Que esto no es nuevo lo muestra claramente la lectura de Quintiliano, en la medida en que

${ }^{36}$ Cf. I.O. XI 3,15-17 y 18-64.

37 J. L. Davitz, The Communication of Emotional Meaning, Nueva York 1964.

${ }^{38}$ Cf. ibid. XI 3,63-65, donde se habla de la adecuación de la voz a las distintas emociones.

${ }_{39}$ T. Kowzan, o.c., pp. 38-9.

${ }^{40}$ Cf. G. L. Trager, "Paralanguage: a first Approximation», en D. Hymes (ed.), Language in Culture and Society, Nueva York 1958, pp. 274-288.

4 Cf. J. Lyons, Semantics, Cambridge 1977, p. 65. 
a cada emoción particular se asigna una base correspondiente para ser asociada por el sujeto a una forma determinada de voz. Un ejemplo ilustrativo nos lo proporciona el siguiente pasaje: Itaque laetis in rebus plena et simplex et ipsa quodam modo hilaris fluit; at in certamine erecta totis uiribus et uelut omnibus neruis intenditur. Atrox in ira et aspera ac densa et respiratione crebra: neque enim potest esse longus spiritus cum immoderate effunditur. Paulum in inuidia facienda lentior, quia non fere ad hanc nisi inferiores confugiunt; at in blandiendo, fatendo, satisfaciendo, rogando lenis et summissa (I.O. XI 3,63). En este punto los principales textos que el autor latino dedica al tema nos producen la impresión de que los términos descriptivos empleados para caracterizar estados psicológicos - del tipo expresivo o emotivo - no constituyen, de hecho, calificaciones arbitrarias o subjetivas, sino que, por el contrario, la expresión de actitudes o de sensaciones está estrechamente codificada en lo que concierne a los indicadores de la voz, aspecto que desde antiguo constituye uno de los objetivos específicos del arte del actor, y que ha sido puesto de relieve por la Semiótica Teatral.

La agudeza e intuición que venimos observando en Quintiliano se pone igualmente de manifiesto al examinar sus consejos sobre el gestus que, como dijimos anteriormente, se corresponde con la actual "cinésica", bajo la que se engloban sistemas de signos como los mímicos, los gestuales y los proxémicos. Así, sus palabras, en las que está constantemente relacionado el gesto con los rasgos vocálicos ${ }^{42}$, vuelven a recordar a Kowzan, autor al que se le atribuye el logro de la puesta en relieve de las relaciones transistémicas en la representación teatral. En el rétor, el tratamiento del gesto está plagado de innumerables comparaciones con la actividad teatral, hasta el punto de que en ocasiones se podría creer que estamos ante un texto dedicado específicamente a la puesta en escena teatral. El gesto es considerado como un auténtico lenguaje según se lee en el pasaje siguiente: Is quantum habeat in oratore momenti satis uel ex eo patet, quod pleraque etiam citra uerba significat (I.O. XI $3,65)^{43}$, aspecto éste recogido por la moderna Semiótica bajo la denominación de "comunicación no verbal»" ${ }^{44}$ La misma idea se repite a lo largo de los capítulos en los que se ocupa de este sistema de signos:

42 I.O. XI 3,65 De gestu prius dicam, qui et ipse uoci consentit et animo cum ea simul paret.

${ }^{43} \mathrm{La}$ preocupación y minuciosidad con la que Quintiliano estudia la fisonomía en las descripciones se observa también en Cicerón,' tanto en el De orat. como en los discursos o los tratados; cf. Leg. I 26, Rep. II 7, Diu. I 79 y II 86-89, Nat. deor. II 42, Fat. IV 7 y V 9.

4 Véase J. Ruesch y W. Kees, Non-Verbal Communication, Los Angeles 1956. 
los gestos son analizados pro sermone, unidos frecuentemente a los verbos significare y ostendere ${ }^{45}$. De todos los movimientos comprendidos bajo la designación general de "gesto", es recurrente la insistencia en la necesidad de que éstos sean puestos en interrelación y estén coordinados, pues, en su opinión, decor quoque a gestu atque motu uenit (I.O. XI $3,67)$, y, al igual que más arriba al hablar del empleo de la voz, se subraya el hecho de que los signos gestuales deben adecuarse al sentido de lo expresado mediante las palabras y deben ser traductores de sentimientos ${ }^{46}$.

A la hora de separar los elementos que intervienen en el gestus, comienza Quintiliano señalando la importancia de la cabeza para la expresión $^{47}$ y a continuación, en un pasaje muy significativo, enumera una serie de posiciones corporales que parecen estar unidas a significados precisos: significat uero plurimis modis. Nam praeter adnuendi, renuendi, confirmandique motus sunt at uerecundiae et dubitationis et admirationis et indignationis noti et communes omnibus... Dominatur autem maxime uultus... hic est saepe pro omnibus uerbis (I.O. XI 3, 70-72). Cuando hace referencia a la relevancia de la cabeza la comparación empleada es la de los comediantes y sus máscaras, más exactamente las utilizadas en la comedia que, a través de unos rasgos estereotipados o hipercodificados, sugerian unos estados de ánimo bien definidos, sin que esto signifique excluir la posible influencia en el rétor de algún tratado de fisonomía, sobre todo si pensamos en el metódico proceder al enumerar y analizar los elementos que se encuentran en la cabeza ${ }^{48}$.

El objetivo que nos hemos propuesto en estas páginas se vería desbordado ampliamente si nos detuviéramos en el análisis puntual de las numerosas observaciones de Quintiliano y las propuestas análogas de la Semiótica Teatral. Pese a ello, no queremos dejar de mencionar algunas de las reflexiones que, en nuestra opinión, son suficientemente explícitas del paralelismo existente ente sus ideas y algunas de las tesis defendidas por la moderna disciplina. Así, por ejemplo, nos parece especialmente significativo el empleo de la denominación indices, con un valor plenamente semiótico, al referirse a las lágrimas con las siguientes palabras: quin etiam lacrimas iis natura mentis indices dedit, quae aut erumpunt dolore aut laetitia manant (I.O. XI 3,75). Igualmente notable es su afirma-

${ }^{45}$ Cf. I.O. XI 3,9; ibid. $65,68,70,72,80,87,97,105$.

46 I.O. XI 3,106 y 116.

47 I.O. XI 3,68.

48 Indiscutiblemente Quintiliano trabajó con distintas fuentes, además de los citados Nigidio y Plocio; cf. I.O. XI 3,106 ueteres artifices... adiecerunt; XII 3,73 artifices pronuntiandi; XI 3,112 artifices... uetant. 
ción sobre la importancia que tienen las manos en la representación, sin las cuales -escribe - trunca esset actio ac debilis (I.O. XI 3,85); hay que señalar que no se trata de meros signos de apoyo, pues a continuación Quintiliano las califica como un auténtico lenguaje: nam ceterae partes loquentem adiunant, hae, prope est ut dicam, ipsae locuntur (ibid.). Llega incluso a afirmar algo que aún hoy día es objeto de disputas, a saber, la posible universalidad de estos lenguajes, carácter del que el rétor se declara abierto partidario: Non in demonstrandis locis atque personis aduerbiorum atque pronominum optinent uicem? - ut in tanta per omnis gentes nationesque linguae diuersitate hic mihi omnium hominum communis sermo uideatur (I.O. XI 3,87), afirmación que por otra parte, no debe extrañar puesto que este autor pertenece a una cultura en la que, como se ha dicho, el gesto es rey, sin embargo no deja de sorprender la extremada precisión en el análisis de los distintos significados expresados mediante el empleo de las manos ${ }^{49}$.

Sin necesidad de extender el tratamiento pormenorizado de los diferentes puntos comentados por Quintiliano, a juzgar por lo anteriormente expuesto, creemos que no resulta descabellado admitir la existencia en la antigüedad de una teoría específica sobre la puesta en escena, tanto oratoria como teatral, y en consecuencia, la de unos códigos perfectamente fijados y conocidos por los destinatarios de la comunicación, teoría que, en ausencia de las obras que debieron existir, aún es rastreable a través de los tratados de Retórica.

Sin haber sido nuestro propósito analizar exhaustivamente las referencias que hace el rétor al complicado proceso de la representación, parece evidente que, si no todos, al menos muchos de los sistemas de signos asignados por la moderna Semiótica a la "puesta en escena» en general ya habían sido mencionados y estudiados por la Retórica antigua, como la lectura de Quintiliano pone de relieve. Con respecto a los datos extraídos en el análisis de la Institutio Oratoria, y en relación con el debate planteado sobre si las reflexiones del autor conciernen a la comedia o a la tragedia ${ }^{50}$, creemos que lo que se desprende con claridad

49 Los gestos que enumera Quintiliano han sido objeto de numerosos estudios; especialmente interesantes son los de B. Warnecke, "Die Gebärden und Mimik der römischen Schauspieler", Neue Jahrb. für Klassische Altertum 13, 1910, pp. 590-594; G. Neumann, Gesten und Gebärden in der griechischen Kunst, Berlin 1965, у B. A. Taladoire, Commentaire sur la mimique et l'expression corporelle du comédien romain, Montpellier 1951.

so B. A. Taladoire, o.c., p. 122, opina que la Institutio Oratoria concierne más a la tragedia que a la comedia, más a la stataria que a la motoria, y más a esta última que a la simple farsa, al ballet o al mimo; por el contrario, A. Pociña, o.c., p. 105 , piensa que Quintiliano presta una mayor atención al actor cómico que al trágico. 
es la existencia de diversos modos de representación adecuados a los distintos géneros teatrales. Por otra parte, y sin olvidar que éste no era el objetivo específico de Quintiliano, no se puede dejar de señalar el profundo sentido plástico y teatral que pone de manifiesto al tratar, entre otros, temas como el del cultus $^{51}$ en la actio, es decir, vestido, peinado, calzado, etc..., considerados todos ellos como auténticos connotadores de la distinción, la elegancia y la virilidad, hasta tal extremo que Cousin ha podido escribir, con razón, que nos parece estar escuchando a un presentador de alta costura ${ }^{52}$.

Tras lo anteriormente expuesto pensamos que es conveniente insistir en la necesidad de estudiar y recuperar las enseñanzas de la antigua $\mathrm{Re}$ tórica en toda una serie de temas que hoy día son presentados con aspecto de novedad y originalidad por la investigación más reciente. En este sentido el caso de Quintiliano, cuyo interés e intuición ya han sido reconocidos en campos como los de la Pedagogía o la Literatura Comparada ${ }^{53}$, constituye un claro ejemplo de lo que una epistemología e historiografia de la ciencia preocupada por rastrear los precedentes de los conceptos teóricos actuales puede conseguir. En el ámbito concreto de los estudios teatrales, las observaciones realizadas por el rétor latino sobre la teoría general de la representación, que hemos ido comentando, permiten considerarlo como un antecedente de lo que en la actualidad se conoce como Semiótica Teatral.

Leonor Perez Gómez

Sobre la relación de la obra del rétor con el teatro de su tiempo véase $\mathbf{J}$. W. Basore, "Quintilian on the status of the later comic stage", en TAPhA 40, 1909, pp. 21-22.

sI Cf. I.O. XI 3,137.

52 J. Cousin, Institution Oratoire, XI, París 1979, p. 174.

53 Véase E. Bolaffi, La critica filosofica e letteraria in Quintiliano, Bruselas 1958, p. 10, y M. Dolç, M. Fabio Quintiliano. Institutio Oratoria. Libro Décimo, Barcelona 1947 , p. 50. 